\title{
Scaling and Asymptotic Scaling in the SU(2) Gauge Theory
}

\author{
J. FINGBERG ${ }^{2}$, U. M. HELLER ${ }^{b}$ and F. KARSCH ${ }^{\text {a }} c$

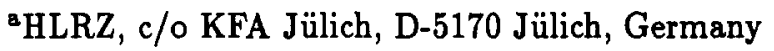 \\ bSCRI, The Florida State University, Tallahassee, FL 32306-44052 U.S.A. \\ 'Fakultät für Physik, Universität Bielefeld, D-4800 Bielefeld 1, Germany
}

We present results from a large volume study of the finite temperature deconfinement phase transition. The questions of scaling and asymptotic scaling are addressed. We find that an extrapolation of the critical temperature and the string tension to the continuum limit is possible when an effective coupling defined in terms of the plaquette operator is used.

\section{INTRODUCTION}

First results for the deconfinement temperature [1], all in the region around $200 \mathrm{MeV}$, were promising but very soon it became clear that larger lattices are required. Even advanced studies of the heavy quark potential on large lattices [2-4] showed that asymptotic scaling is not yet restored at the values of the bare coupling constant presently accessible.

Recent investigations of the finite temperature deconfinement transition using finite-size scaling techniques showed that finite spatial size corrections are well under control and that reliable methods to determine the critical coupling [5-7] are available. We have extended these studies to smaller lattice spacings using lattices of size $N_{\sigma}^{3} \times N_{\tau}$ with $N_{\tau}=8$ and 16 . This makes it possible to study scaling of the dimensionless ratio $T_{c} / \sqrt{\sigma}$ and the approach to asymptotic scaling in different extrapolation schemes.

\section{FINITE-SIZE SCALING AND THE CONTINUUM LIMIT}

Finite-size scaling techniques can be used to relate the critical behaviour observed on finite lattices to the corresponding continuum field theory.

The singular part of the free energy density for a continuum field theory with a simple critical point in a finite volume can be written in terms of lattice variables as the product of a scaling function $Q_{f}$, and a factor $y$ which is the ratio of the spatial and temporal lattice size [8]. $Q_{f}$, depends on the reduced temperature $t$ and the external magnetic field strength $h$ in form of a thermal and a magnetic scaling field $g_{t}$ and $g_{h}$.

$$
f_{s}\left(t, h ; N_{\sigma} ; N_{\tau}\right)=y^{-3} Q_{f}\left(g_{t} y^{\frac{2}{\nu}}, g_{h} y^{\frac{e+\gamma}{\nu}}\right)
$$

The Binder cumulant $g_{4}$ is defined through the free energy density as

$$
\begin{aligned}
g_{4} & =\left.\frac{\partial^{4} f_{s}}{\partial h^{4}}\right|_{h=0} /\left(\left.\frac{\partial^{2} f_{b}}{\partial h^{2}}\right|_{h=0}\right)^{2} y^{3} \\
& =\left\langle L^{4}\right) /\left\langle L^{2}\right\rangle^{2}-3 .
\end{aligned}
$$

On a finite lattice, $g_{4}$ is measured by the second and fourth moment of the Polyakov loop operator. The cumulant $g_{4}$ depends only on $g_{t} y^{1 / \nu}$ and for small values of the reduced temperature one can use a linear expansion.

$g_{4}\left(g_{t} y^{1 / \nu}\right)=g_{4,0}+g_{4,1} t y^{1 / \nu}$

For fixed $N_{\tau}$ and varying $N_{\sigma}$ the critical coupling for infinite volume $\beta_{c, \infty}$ can be determined from the fixed point $g_{4}(t=0)$. For a fixed value of $y$, varying $N_{\tau}$ and $N_{\sigma}$ accordingly, the critical coupling can be extracted from the shift $\Delta \beta$ which is necessary to overlay two curves of $g_{4}$.

$$
\begin{aligned}
& g_{4}\left(\beta-\Delta \beta ; N_{\sigma} ; N_{\tau}\right)=g_{4}\left(\beta ; 2 N_{o} ; 2 N_{\tau}\right) \\
& \beta_{c, \infty}\left(2 N_{\tau}\right)=\beta_{c, \infty}\left(N_{\tau}\right)+\Delta \beta
\end{aligned}
$$

The continuum limit can be found by keeping $y$ fixed and increasing $N_{\tau}$. The numerical results for $g_{4}$ are shown in Figs. 1 and 2. The continuous curves were obtained by the density of states 


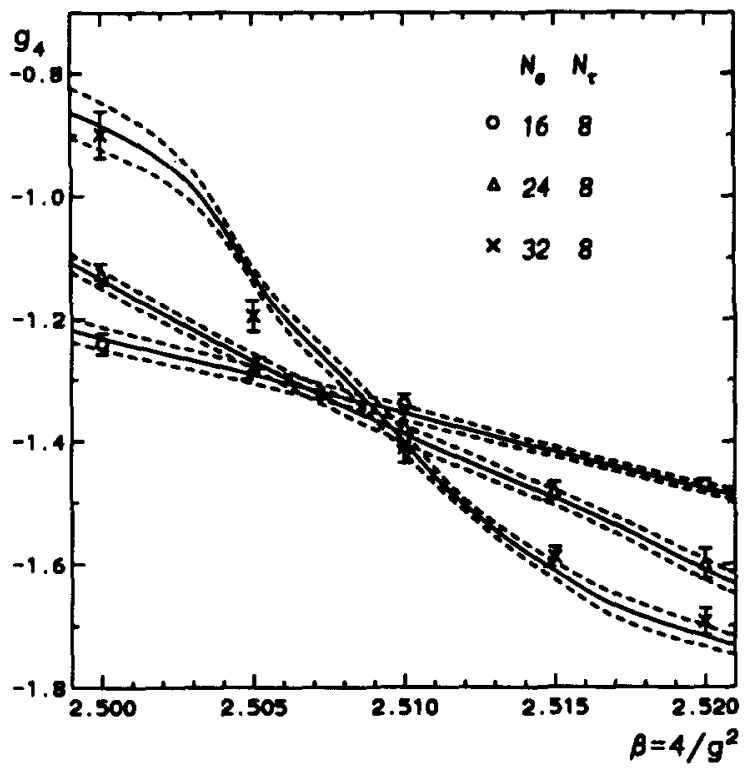

Figure 1. The cumulant $g_{4}$ for $N_{T}=8$.

method. Using the fixed point method for $N_{\tau}=8$ and the shift for $N_{\tau}=16$ we obtain the following values for the critical couplings

$$
\beta_{c, \infty}=\left\{\begin{array}{ll}
2.5115(40), & N_{\tau}=8 \\
2.7395(100), & N_{\tau}=16
\end{array} .\right.
$$

\section{SCALING AND ASYMPTOTIC SCALING}

The scaling behaviour near the continuum limit is controlled by the asymptotic freedom property of QCD which implies scaling of dimensionless ratios of physical quantities.

For $\mathrm{SU}(\mathrm{N})$ gauge theories the ratio $V_{c}(R) / \sqrt{\sigma}$ scales as a function of $R \sqrt{\sigma}$ for $\beta \geq 2.4,6.0$ for $N=2,3[2,3]$.

A comparison with string tension data [8] shown in Fig. 3 reveals scaling of $T_{c} / \sqrt{\sigma}$ in the region $4 \leq N_{\tau} \leq 16$ for $\mathrm{SU}(2)$ and in the region $8 \leq N_{\tau} \leq 14$ for $\mathrm{SU}(3)$. By averaging over the scaling region we obtain the continuum result

$\frac{T_{c}}{\sqrt{\sigma}}=\left\{\begin{array}{ll}0.69 \pm 0.02, & S U(2) \\ 0.56 \pm 0.03, & S U(3)\end{array}\right.$.

The fact that we observe scaling but not asymp-

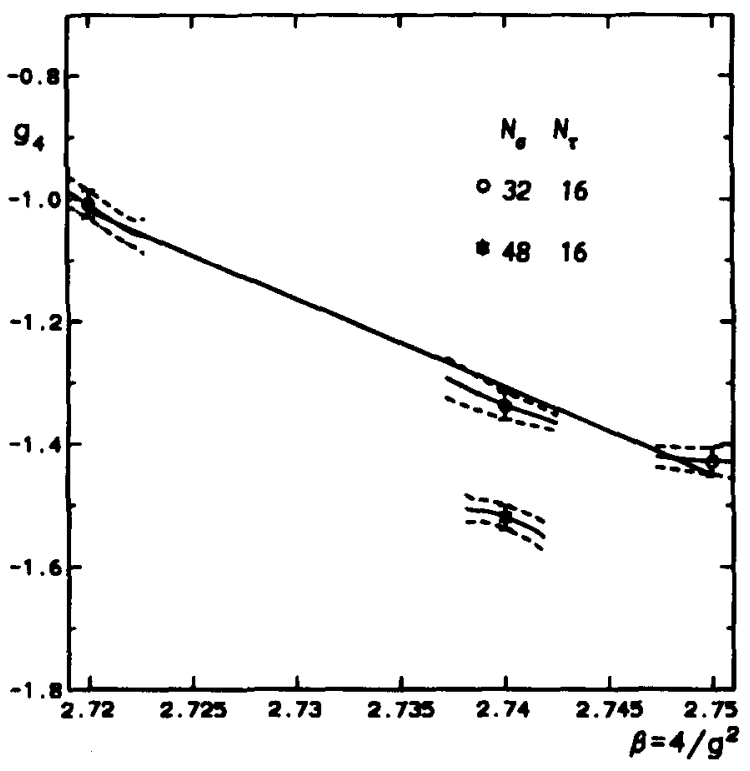

Figure 2. The cumulant $g_{4}$ for $N_{\tau}=16$.

totic scaling indicates the presence of universal scaling violating terms which can be incorporated by a redefinition of the bare coupling $\beta$. Following Parisi [9], an effective coupling scheme which has been successfully applied [10] can be defined in terms of the plaquette operator.

$\beta_{E}=\frac{N^{2}-1}{4\left(1-\left\langle U_{p}\right)\right)}$

Various other definitions giving similar results are possible $[11,12]$. In Figs. 4 and 5 we show that violations of asymptotic freedom are strongly reduced in the effective coupling scheme. In the continuum limit scaling violating terms are expected to be $\mathcal{O}(1 / \ln a)$. The data are not (yet) sensitive to this logarithmic correction. A linear extrapolation to $a=0$ yields

$$
\frac{T_{c}}{\Lambda_{\overline{\mathrm{MS}}}}= \begin{cases}1.23 \pm 0.11, & S U(2) \\ 1.03 \pm 0.19, & S U(3) .\end{cases}
$$

The corresponding extrapolation for the string tension, shown in Fig. 5, results in

$$
\frac{\sqrt{\sigma}}{\Lambda_{\overline{M S}}}= \begin{cases}1.79 \pm 0.12, & S U(2) \\ 1.75 \pm 0.20, & S U(3) .\end{cases}
$$




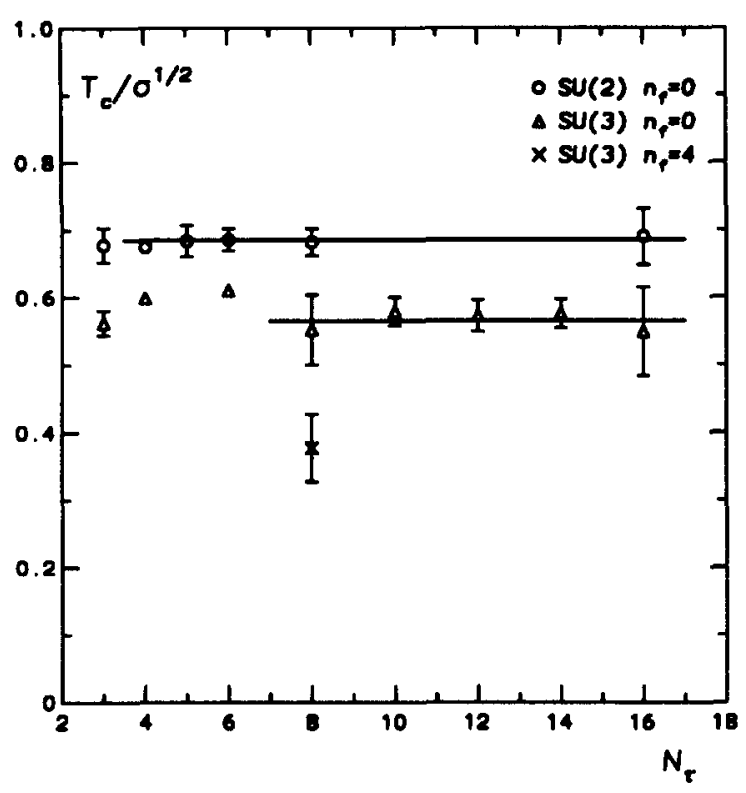

Figure 3. The critical temperature in units of the square root of the string tension.

We stress once more that the linear extrapolation eventually should turn to a logarithmic one at even smaller values of the lattice spacing.

\section{Conclusion}

The ratio $T_{c} / \sqrt{\sigma}$ scales for $\beta \geq 2.3$ over a range, where the lattice spacing varies by a factor of 4 and the smallest lattice spacing we reached was $a=0.09 / \sqrt{\sigma}$.

A comparison between the bare and an effective coupling scheme shows that violations of asymptotic scaling are strongly reduced in the latter case. Converting our continuum result for the string tension to a value of the $\Lambda$-parameter and comparing with other recent results we see that the numbers do not differ significantly. The last value in $\mathrm{Eq} .12$ has been obtained by using the perturbative form of the running coupling [13].

$$
\Lambda_{\overline{\mathrm{MS}}}^{S U(2)} / \sqrt{\sigma}= \begin{cases}0.559(37) & \\ 0.617(55), & \text { Ref.[2] } \\ 0.478, & \text { Ref.[12] }\end{cases}
$$

$$
\Lambda_{\overline{\mathrm{MS}}}^{S U(3)} / \sqrt{\sigma}= \begin{cases}0.571(37) & \\ 0.558_{-0.007}^{+0.017}, & \operatorname{Ref} .[3] \\ 0.581(45), & \operatorname{Ref} .[14] \\ 0.532, & \operatorname{Ref} .[11]\end{cases}
$$

These results give further confidence that an extrapolation to the continuum limit $a=0$ is within reach for the pure gauge theory. For the future systematic errors possibly present in the applied methods have to be further reduced. Then a remaining challenging task is the extension of these methods to full QCD including dynamical fermions.

\section{REFERENCES}

1 J. Kuti, J. Polónyi and K. Szlachányi, Phys. Lett. 98B (1980) 199, J. Engels, F. Karsch and H. Satz, Phys. Lett. 101B (1981) 89, K. Kajantie, C. Montonen and E. Pietarinen, Z. Phys. C9 (1981) 253.

2 S. P. Booth et al. (the UKQCD Collaboration), Phys. Lett. 275B (1992) 424, Liverpool preprint LTH284.

3 G. S. Bali and K. Schilling, Wuppertal preprint WUB 92-02 and WUB 92-29.

4 C. Michael, Phys. Lett. 283B (1992) 103.

5 J. Engels, J. Fingberg, and M. Weber, Nucl. Phys. B332 (1990) 737.

6 J. Engels, J. Fingberg and D. Miller, to appear in Nucl. Phys. B (1992).

7 J. Engels, J. Fingberg and V. K. Mitrjushkin, Bielefeld preprint BI-TP 92/22.

8 J. Fingberg, U. Heller and F. Karsch, Bielefeld preprint BI-TP $92 / 26$ and references within.

9 G. Parisi, Proceedings of the $X X^{t h}$ Conference on High Energy Physics, Madison 1980.

10 F. Karsch and R. Petronzio, Phys. Lett. 139B (1984) 403.

11 A. X. El-Khadra et al., FERMILAB-PUB91/354-T, G. P. Lepage and P. B. Mackenzie, FERMILAB-PUB-91/355-T.

12 M. Lüscher, R. Sommer, P. Weisz and U. Wolff, Cern preprint CERN-TH 6566/92.

13 A. Billoire, Phys. Lett. 104B (1981) 472.

14 S. P. Booth et al. (the UKQCD Collaboration), Liverpool preprint LTH285. 

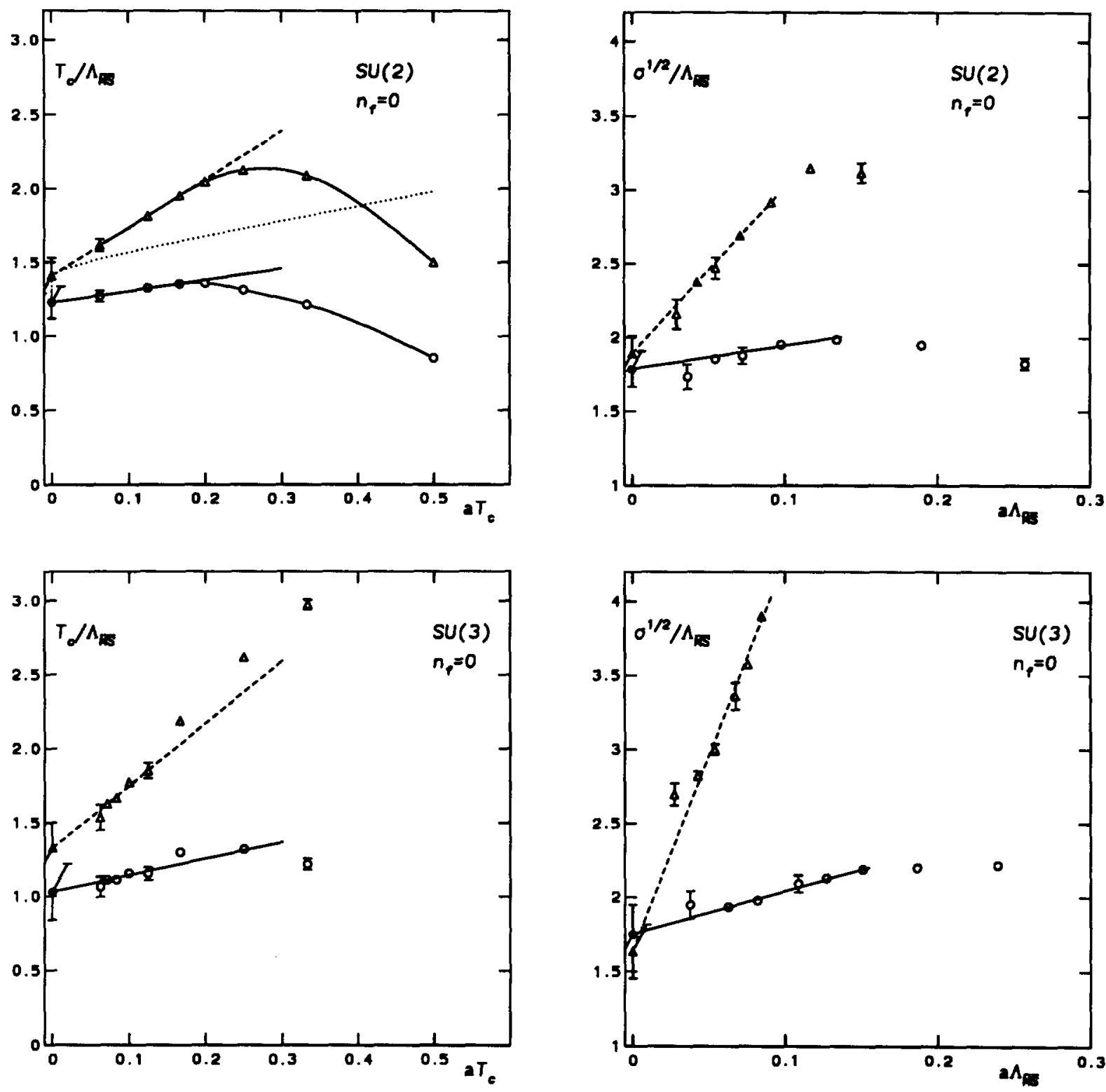

Figure 4. Extrapolation of the critical temperature in the bare (triangles) and the effective (circles) coupling scheme.

Figure 5. Extrapolation of the string tension in the bare (triangles) and the effective (circles) coupling scheme. 